MSC 60H30, 60H10

DOI: $10.14529 / \mathrm{mmp} 190212$

\title{
STOCHASTIC INCLUSIONS WITH FORWARD MEAN DERIVATIVES HAVING DECOMPOSABLE RIGHT-HAND SIDES
}

\author{
A.V. Makarova, N.E. Zhukovsky and Y.A. Gagarin Air Force Academy, Voronezh, \\ Russian Federation, allagm@mail.ru
}

In this paper, we prove a theorem on the existence of solutions for stochastic differential inclusions given in terms of the forward mean derivatives and the quadratic mean derivatives. These derivatives present information on the drift and the diffusion coefficient, respectively. The forward mean derivatives were introduced by E. Nelson for the needs of the so-called stochastic mechanics (a version of quantum mechanics), while the quadratic mean derivatives were introduced by Yu.E. Gliklich and S.V. Azarina. In the case of both the forward mean derivatives and the quadratic mean derivatives, we assume that the righthand side is set-valued and lower semi-continuous, but not necessarily convex. Instead of this, we assume that the right-hand side is decomposable. Such inclusions naturally arise in many models of physical processes.

Ключевые слова: mean derivatives; decomposable set-valued mappings; differential inclusions.

Dedicated to Yu. Gliklikh's 70-th bithday.

\section{Introduction}

The notion of mean derivative was introduced by Edward Nelson [1-3] for the needs of stochastic mechanics (a version of quantum mechanics). Then it was found that the equations in mean derivatives take place also in many other branches of science (mechanics, hydrodynamics, Navier-Stokes vortices, gauge fields, economics, etc.).

A new mean derivative called quadratic is introduced in [4]. The derivative gives information on the diffusion coefficient of the process. Using Nelson's and quadratic mean derivatives together, we can recover the process from its mean derivatives under some additional hypotheses.

Note that a lot of models of various physical, economical etc. processes are based on equations and inclusions with mean derivatives. Also, the inclusions play important role in the optimal control problems.

We investigate inclusions with forward mean derivatives having lower semi-continuous right-hand sides. The images of points are not necessary convex sets. Instead of this, we suppose that the right-hand sides are decomposable. This property provides serious modification of all proofs and constructions. We obtain an existence of solution theorem for such inclusions. Note that the arguments are seriously different form those in [13].

Let $S(n)$ be the space of symmetric $n \times n$ matrices, $S_{+}(n)$ be the subset of positive defined symmetric matrices, and $\bar{S}_{+}(n)$ be its closure, i.e. the set of positive semi-definite symmetric matrices.

\section{Preliminaries on Mean Derivatives}

Let $\xi(t)$ be a given stochastic process with values in $\mathbb{R}^{n}$ on certain probability space $(\Omega, \mathcal{F}, \mathrm{P})$. Suppose that the element $\xi(t)$ belongs to the functional space $L_{1}$ for every 
$t$. Denote by $\mathcal{P}_{t}^{\xi}$ the $\sigma$-subalgebra of $\mathcal{F}$ that is generated by preimages of Borel sets in $\mathbb{R}^{n}$ under all mappings $\xi(s): \Omega \rightarrow \mathbb{R}^{n}, 0<s<t$. By $E\left(\cdot \mid \mathcal{P}_{t}^{\xi}\right)$ we denote the conditional expectation with respect to $\mathcal{P}_{t}^{\xi}$. Following Nelson [1-3], we call $\mathcal{P}_{t}^{\xi}$ the past of the process $\xi(t)$.

Definition 1. [1,4] The forward mean derivative relative to the past ( $\mathcal{P}$-mean derivative) $D^{\mathcal{P}} \xi(t)$ of $\xi(t)$ at the time $t$ is $L_{1}$-random element of the form

$$
D^{\mathcal{P}} \xi(t)=\lim _{\triangle t \rightarrow+0} E\left(\frac{\xi(t+\triangle t)-\xi(t)}{\triangle t} \mid \mathcal{P}_{t}^{\xi}\right),
$$

where the limit is assumed to exist in $L_{1}$, and $\triangle t \rightarrow+0$ means that $\triangle t$ tends to 0 and $\triangle t>0$.

The properties of conditional expectation [6] give that $D \xi(t)$ can be represented as compositions of $\xi(t)$ and Borel measurable vector fields (regressions)

$$
a(t, x)=\lim _{\Delta t \rightarrow+0} E\left(\frac{\xi(t+\Delta t)-\xi(t)}{\Delta t} \mid \xi(t)\right)=x
$$

on $\mathbb{R}^{n}$. This means that $D \xi(t)=a(t, \xi(t))$.

Following $[4,8]$, we introduce a new mean derivative $D_{2}$, called quadratic relative to the past, that differentiates a $L_{1}$ random process $\xi(t), t \in[0, T]$, according to the rule

$$
D_{2}^{\mathcal{P}} \xi(t)=\lim _{\triangle t \rightarrow+0} E_{\mathcal{P}}^{\xi}\left(\frac{(\xi(t+\triangle t)-\xi(t)) \otimes(\xi(t+\triangle t)-\xi(t))}{\triangle t} \mid \mathcal{P}_{t}^{\xi}\right),
$$

where the limit is assumed to exist in $L_{1}, \Delta t \rightarrow+0$ means that $\Delta t$ tends to 0 and $\Delta t>0$, and $\otimes$ denotes the tensor product in $\mathbb{R}^{n}$. Here $(\xi(t+\triangle t)-\xi(t))$ is considered as a column vector in $\mathbb{R}^{n}$ while $(\xi(t+\triangle t)-\xi(t))^{*}$ is a row vector (transposed or conjugate vector), and the limit is supposed to exist in $L_{1}(\Omega, \mathcal{F}, \mathrm{P})$.

We emphasize that the matrix product of a column on the left and a row on the right is a matrix. It is shown that $D_{2} \xi(t)$ is a symmetric positive semi-definite matrix function on $[0, T] \times \mathbb{R}^{n}$.

Consider the Banach space of continuous curves in $\mathbb{R}^{n}$ given on $[0, T]: \tilde{\Omega}=$ $C^{0}\left([0, T], \mathbb{R}^{n}\right)$ with usual uniform norm and the $\sigma$-algebra $\tilde{\mathcal{F}}$ generated by cylinder sets. By $\mathcal{P}_{t}$ we denote the $\sigma$-subalgebra of $\mathcal{F}$ generated by cylinder sets with bases over $[0, t] \subset[0, T]$. Recall that $\tilde{\mathcal{F}}$ is the Borel $\sigma$-algebra on $\tilde{\Omega}$. Let $a:[0, T] \times \tilde{\Omega} \rightarrow \mathbb{R}^{n}$ and $\alpha:[0, T] \times \tilde{\Omega} \rightarrow \bar{S}_{+}(n)$ be measurable mappings.

The equation with $\mathcal{P}$-mean derivatives is a system of the form

$$
\left\{\begin{array}{l}
D^{\mathcal{P}} \xi(t)=a(t, \xi(\cdot)) \\
D_{2}^{\mathcal{P}} \xi(t)=\alpha(t, \xi(\cdot))
\end{array}\right.
$$

Definition 2. [5] We say that (4) on $\mathbb{R}^{n}$ has a solution on $[0, T]$ with initial condition $\xi(0)=\xi_{0}$ if there exists a probability space $(\Omega, \mathcal{F}, \mathrm{P})$ and a process $\xi(t)$ given on $(\Omega, \mathcal{F}, \mathrm{P})$ and taking values in $\mathbb{R}^{n}$ such that $\xi(0)=\xi_{0}$ and for almost all $t \in[0, T]$ equation (4) is satisfied $\mathrm{P}$ a.s. by $\xi(t)$.

Let $\Xi:[0, T] \times \tilde{\Omega} \rightarrow Z$ be a mapping to some metric space $Z$. Below we often suppose that such mappings with various spaces $Z$ satisfy the following condition. 
Condition 1. $\Xi\left(t, x_{1}(\cdot)\right)=\Xi\left(t, x_{2}(\cdot)\right)$ for each $t \in[0, T]$, if the curves $x_{1}(\cdot), x_{2}(\cdot) \in \tilde{\Omega}$ coincide for $0 \leq s \leq t$.

Remark 1. Note that the fact that a mapping $\Xi$ satisfies Condition 1 is equivalent to the fact that $\Xi$ for each $t$ is measurable with respect to Borel $\sigma$-algebra in $Z$ and $\mathcal{P}_{t}$ in $\tilde{\Omega}$ [11].

Lemma 1. [5] For a continuous (measurable, smooth) mapping $\alpha:[0, T] \times \tilde{\Omega} \rightarrow$ $S_{+}(n)$ satisfying Condition 1, there exists a continuous (measurable, smooth, respectively) mapping $A:[0, T] \times \tilde{\Omega} \rightarrow L\left(\mathbb{R}^{n}, \mathbb{R}^{n}\right)$ that satisfies Condition 1 and such that $\alpha(t, x(\cdot))=$ $A(t, x(\cdot)) A^{*}(t, x(\cdot))$ for each $(t, x(\cdot)) \in \mathbb{R} \times \tilde{\Omega}$.

The proof of Lemma 1 can be found in [5, Lemma 1].

Theorem 1. [4] Let $a:[0, T] \times \tilde{\Omega} \rightarrow \mathbb{R}^{n}$ and $\alpha:[0, T] \times \tilde{\Omega} \rightarrow S_{+}(n)$ be jointly continuous in $t, x(\cdot)$ and satisfy Condition 1 . Also, suppose that the estimates

$$
\begin{gathered}
\operatorname{tr} \alpha(t, x(\cdot))<\Theta(1+\|x(\cdot)\|)^{2}, \\
\|a(t, x(\cdot))\|<\Theta(1+\|x(\cdot)\|)
\end{gathered}
$$

take place for some $\Theta>0$. Then for every initial condition $\xi_{0} \in \mathbb{R}^{n}$ equation (4) has a solution that is well-defined on the entire interval $[0, T]$.

\section{Main Result}

Sometimes we use a modification of the system of arguments from [12]. If the values of a lower semi-continuous set-valued mapping (generally speaking) are not convex, then the mapping can not have continuous selectors. Hence, the following construction is often very useful.

Definition 3. [9] Let $E$ be a separable Banach space. A non-empty set $\mathcal{M} \subset L^{1}([0, l] ; E)$ is called decomposable, if $f \cdot \chi_{\mathfrak{M}}+g \cdot \chi_{[0, T] \backslash \mathfrak{M}} \in \mathcal{M}$ for all $f, g \in \mathcal{M}$ and for every measurable subset $\mathfrak{M}$ in $[0, T]$, where $\chi$ is the characteristic function of the corresponding set.

The reader can find more details about decomposable sets in $[9,10]$.

Theorem 2. (The Bressan - Colombo Theorem [9]) Let $(\Omega, d)$ be a separable metric space, $X$ be a Banach space, and $(J, \mathcal{A}, \mu)$ be a measurable space with a $\sigma$-algebra $\mathcal{A}$ and a non-atomic measure $\mu$ such that $\mu(J)=1$. Consider the space $Y=L_{X}^{1}(J, \mathcal{A}, \mu)$ of integrable mappings from $(J, \mathcal{A}, \mu)$ into $X$. If a set-valued mapping $F: \Omega \rightarrow Y$ is lower semicontinuous and has close decomposable values, then $F$ has a continuous selector.

Theorem 2 is proved, e.g., in [9, Lemma 9.2]. Recall some facts and notion involved in further considerations. Suppose that $T>0$. In what follows we denote by $\lambda$ the normalized Lebesgue measure on $[0, T]$, i.e. such that $\lambda([0, T])=1$.

Lemma 2. [10] Let $(\Xi, d)$ be a separable metric space, and $X$ be a Banach space. Consider the space $\left.Y=L^{1}(([0, T], \mathcal{B}, \lambda), X)\right)$ of integrable maps from $[0, T]$ into $X$. If a set-valued map $G: \Xi \rightarrow Y$ is lower semicontinuous and has closed decomposable images, then the map has a continuous selector. 
This is a particular case of Bressan - Colombo Theorem 2. Consider set-valued mappings $\boldsymbol{a}(t, x)$ and $\boldsymbol{\alpha}(t, x)$ that send $[0, T] \times \tilde{\Omega}$ to $\mathbb{R}^{n}$ and $\bar{S}_{+}(n)$, respectively, and in addition satisfy Condition 1 . The differential inclusion with forward $\mathcal{P}$-mean derivatives is a system of the form

$$
\left\{\begin{array}{l}
D^{\mathcal{P}} \xi(t) \in \boldsymbol{a}(t, \xi(\cdot)) \\
D_{2}^{\mathcal{P}} \xi(t) \in \boldsymbol{\alpha}(t, \xi(\cdot))
\end{array}\right.
$$

Definition 4. [5] We say that inclusion (7) has a solution with initial condition $\xi_{0} \in \mathbb{R}^{n}$, if there exists a probability space and a stochastic process $\xi(t)$ given on the space and taking values in $\mathbb{R}^{n}$ such that $\xi(0)=\xi_{0}$ and a.s. $\xi(t)$ satisfies inclusion (7).

For simplicity below we consider only solutions with deterministic initial values.

Theorem 3. Let the set-valued fields a and $\boldsymbol{\alpha}$ on $\mathbb{R}^{n}$ be lower semicontinuous and have closed decomposable images of points. Also, suppose that $\boldsymbol{\alpha}$ is positive definite, and the following estimates hold:

$$
\operatorname{tr} \alpha(t, x(\cdot))<\Theta\left(1+\|x(\cdot)\|_{C^{0}}\right)^{2},
$$

and

$$
\|a(t, x(\cdot))\|<\Theta\left(1+\|x(\cdot)\|_{C^{0}}\right)
$$

for all $\alpha(t, x) \in \boldsymbol{\alpha}, a \in \mathbf{a}$ and for some $\Theta>0$, where $\|\cdot\|_{C^{0}}$ is the norm in $C^{0}\left([0, T], \mathbb{R}^{n}\right)$. Then for the initial condition $\xi(0)=\xi_{0}$ inclusion (7) has a solution, which is well-defined on the entire interval $t \in[0, T]$.

Proof. We apply a serious modification of arguments used in [8, Chapter: Mechanical systems with random perturbations]. According to Lemma 2, the set-valued fields a and $\boldsymbol{\alpha}$ have continuous selectors $a$ and $\alpha$, respectively. Construct a sequence of their smooth approximations $a_{k}$ and $\alpha_{k}$ that converge to $a$ and $\alpha$ with respect to the supremum norm. In addition, it is possible to construct $\alpha_{k}$ to be symmetric and positive definite, since we deal with approximations in the space of symmetric matrices, and any positive semi-definite matrix is a limit point of the space of positive definite matrices.

Let $x(\cdot)$ be a continuous curve. Consider the set-valued vector field $\mathbf{a}(t, x(\cdot))$ along $x(\cdot)$. Denote by $\mathcal{P} \mathbf{a}(\cdot, x(\cdot))$ the set of all measurable selectors of $\mathbf{a}(t, x(\cdot))$, i.e. the set of measurable maps $\left\{f: \mathbb{R} \rightarrow \mathbb{R}^{n}: f(x(t)) \in \mathbf{a}(t, x(\cdot))\right\}$. Obviously, all those selectors are integrable on any finite interval in $\mathbb{R}$ with respect to Lebesgue measure, since estimate (9) is satisfied. Denote by $\int \mathcal{P} \mathbf{a}(\cdot, x(\cdot))$ the set of integrals with varying upper limits of those selectors.

In $C^{0}\left([0, T], \mathbb{R}^{n}\right)$, introduce the $\sigma$-algebra $\tilde{\mathcal{F}}$ generated by cylindrical sets. By $\tilde{\mathcal{P}}_{t}$ denote the $\sigma$-algebra generated by cylindrical sets over $[0, t] \subset[0, T]$.

Consider the set-valued mapping $B$ that sends $x(\cdot) \in C^{0}\left([0, T], \mathbb{R}^{n}\right)$ to $\mathcal{P} \mathbf{a}(\cdot, x(\cdot))$. Because of estimate $(9)$, all selectors from $\mathcal{P} \mathbf{a}(\cdot, x(\cdot))$ are integrable (see above), hence $B$ takes values in the space $L^{1}\left(([0, T], \mathcal{B}, \lambda), \mathbb{R}^{n}\right)$. It is known (see, e.g., [10, Section 5.5]) that under the above-mentioned conditions $B: C^{0}\left([0, T], \mathbb{R}^{n}\right) \rightarrow L^{1}\left(([0, T], \mathcal{B}, \lambda), \mathbb{R}^{n}\right)$ is lower semicontinuous, and for any $x(\cdot) \in C^{0}\left([0, T], \mathbb{R}^{n}\right)$ the set $\mathcal{P a}(\cdot, x(\cdot))$ (the image $B(x(\cdot)))$ is decomposable and closed. Therefore, by Lemma $2, B$ has a continuous selector $b: C^{0}\left([0, T], \mathbb{R}^{n}\right) \rightarrow L^{1}\left(([0, T], \mathcal{B}, \lambda), \mathbb{R}^{n}\right)$. 
For any $t \in[0, T]$, we introduce the map $f_{t}: C^{0}\left([0, T], \mathbb{R}^{n}\right) \rightarrow C^{0}\left([0, T], \mathbb{R}^{n}\right)$ that sends a curve $x(\cdot) \in C^{0}\left([0, T], \mathbb{R}^{n}\right)$ to the curve

$$
f_{t}(\tau, x(\cdot))= \begin{cases}x(\tau) & \text { for } \tau \in[0, t] \\ x(t) & \text { for } \tau \in[t, l]\end{cases}
$$

Obviously, the map $f_{t}$ is continuous. Since $f_{t}(\tau, x(\cdot))$ belongs to $C^{0}\left([0, T], \mathbb{R}^{n}\right)$, the curve $b\left(f_{t}(\tau, x(\cdot))\right) \in L^{1}\left(([0, T], \mathcal{B}, \lambda), \mathbb{R}^{n}\right)$ is well defined. By construction, $b\left(f_{t}(\tau, x(\cdot))\right) \in$ $\mathbf{a}(\tau, x(\tau))$ for almost all $\tau \in[0, t]$. Therefore, this selector continuously depends on $t$ in $L^{1}\left(([0, T], \mathcal{B}, \lambda), \mathbb{R}^{n}\right)$.

Introduce the map $a:[0, T] \times C^{0}\left([0, T], \mathbb{R}^{n}\right) \rightarrow \mathbb{R}^{n}$ by the formula

$$
a(t, x(\cdot))=b\left(f_{t}(\tau, x(\cdot))\right)
$$

By construction, this map is continuous jointly for $t \in[0, T]$ and $x(\cdot) \in C^{0}\left([0, T], \mathbb{R}^{n}\right)$. It is obvious that $a\left(t, x_{1}(\cdot)\right)=a\left(t, x_{2}(\cdot)\right)$, if $x_{1}(\cdot)$ and $x_{2}(\cdot)$ coincide on $[0, t]$. This means that $a(t, x(\cdot))$ is measurable with respect to $\tilde{\mathcal{P}}_{t}$ (see Remark 1 ). Taking into account (9), we can easily derive the inequality

$$
\|a(t, x(\cdot))\|=\left\|b\left(f_{t}(\tau, x(\cdot))\right) \leq\right\| \mathbf{a}(\tau, x(\cdot)) \| \leq \Theta\left(1+\|x(\cdot)\|_{C^{0}}\right) .
$$

It is evident that

$$
D^{\mathcal{P}}\left(f_{t}(\tau, \xi(\cdot))\right)=a(t, \xi(\cdot)) \in \mathbf{a}(t, \xi(\cdot)) .
$$

Consider the set-valued vector field $\boldsymbol{\alpha}(t, x(\cdot))$ along a continuous curve $x(\cdot)$. Denote by $\mathcal{P} \boldsymbol{\alpha}(\cdot, x(\cdot))$ the set of all measurable selectors of $\boldsymbol{\alpha}(t, x(\cdot))$ (the set of measurable maps $\left.\left\{f: \mathbb{R} \rightarrow S_{+}(n): f(x(t)) \in \boldsymbol{\alpha}(t, x(\cdot))\right\}\right)$. It is evident that all those selectors are integrable on any finite interval in $\mathbb{R}$ with respect to Lebesgue measure, since estimate (9) is satisfied. Denote by $\int \mathcal{P} \boldsymbol{\alpha}(\cdot, x(\cdot))$ the set of integrals with varying upper limits of those selectors. In $C^{0}\left([0, T], \mathbb{R}^{n}\right)$, introduce the $\sigma$-algebra $\tilde{\mathcal{F}}$ generated by cylindrical sets. By $\tilde{\mathcal{P}}_{t}$ denote the $\sigma$-algebra generated by cylindrical sets over $[0, t] \subset[0, T]$.

Consider the set-valued mapping $\bar{B}$ that sends $x(\cdot) \in C^{0}\left([0, T], \mathbb{R}^{n}\right)$ to $\mathcal{P} \boldsymbol{\alpha}(\cdot, x(\cdot))$. Since all selectors from $\mathcal{P} \boldsymbol{\alpha}(\cdot, x(\cdot))$ are integrable under estimate (8) (see above), $\bar{B}$ takes values in the space $L^{1}\left(([0, T], \mathcal{B}, \lambda), S_{+}(n)\right)$. It is known [10, Section 5.5] that under the above-mentioned conditions $\bar{B}: C^{0}\left([0, T], \mathbb{R}^{n}\right) \rightarrow L^{1}\left(([0, T], \mathcal{B}, \lambda), S_{+}(n)\right)$ is lower semicontinuous, and for any $x(\cdot) \in C^{0}\left([0, T], \mathbb{R}^{n}\right)$ the set $\mathcal{P} \boldsymbol{\alpha}(\cdot, x(\cdot))$, i.e. the image $\bar{B}(x(\cdot))$ is decomposable and closed. Therefore, by Lemma 2, $\bar{B}$ has a continuous selector $\bar{b}: C^{0}\left([0, T], \mathbb{R}^{n}\right) \rightarrow L^{1}\left(([0, T], \mathcal{B}, \lambda), S_{+}(n)\right)$.

Since $f_{t}(\tau, x(\cdot))$ belongs to $C^{0}\left([0, T], \mathbb{R}^{n}\right)$, the curve $\bar{b}\left(f_{t}(\tau, x(\cdot))\right) \quad \in$ $L^{1}\left(([0, T], \mathcal{B}, \lambda), S_{+}(n)\right)$ is well-defined. By construction, $\bar{b}\left(f_{t}(\tau, x(\cdot))\right) \in \boldsymbol{\alpha}(\tau, x(\tau))$ for almost all $\tau \in[0, t]$, and this selector continuously depends on $t$ in $L^{1}\left(([0, T], \mathcal{B}, \lambda), S_{+}(n)\right)$.

Consider the map $\alpha:[0, T] \times C^{0}\left([0, T], \mathbb{R}^{n}\right) \rightarrow S_{+}(n)$ defined by the formula

$$
\alpha(t, x(\cdot))=\bar{b}\left(f_{t}(\tau, x(\cdot))\right) .
$$

By construction, this map is continuous jointly for $t \in[0, T]$ and $x(\cdot) \in C^{0}\left([0, T], \mathbb{R}^{n}\right)$. In addition, it is obvious that $\alpha\left(t, x_{1}(\cdot)\right)=\alpha\left(t, x_{2}(\cdot)\right)$, if $x_{1}(\cdot)$ and $x_{2}(\cdot)$ coincide on $[0, t]$. This means that $a(t, x(\cdot))$ is measurable with respect to $\tilde{\mathcal{P}}_{t}$ (see Remark 1 ). 
It is evident that

$$
D_{2}^{\mathcal{P}}\left(f_{t}(\tau, \xi(\cdot))\right)=\alpha(t, \xi(\cdot)) \in \boldsymbol{\alpha}(t, \xi(\cdot)) .
$$

By Lemma 1, there exists a continuous mapping $A:[0, T] \times C^{0}\left([0, T], \mathbb{R}^{n}\right) \rightarrow L\left(\mathbb{R}^{n}, \mathbb{R}^{n}\right)$ that satisfies Condition 1 and such that $\alpha(t, x(\cdot))=A(t, x(\cdot)) A^{*}(t, x(\cdot))$ for each $(t, x(\cdot)) \in$ $\mathbb{R} \times C^{0}\left([0, T], \mathbb{R}^{n}\right)$.

Taking into account (8), we can easily derive the inequality

$$
\|A(t, x(\cdot))\| \leq \Theta\left(1+\|x(\cdot)\|_{C^{0}}\right) .
$$

Now the couple $a(t, x(\cdot))$ and $A(t, x(\cdot))$ satisfies all conditions of Theorem $1[11$, Section III.2]. Hence, the stochastic differential equation

$$
\xi(t)=\xi_{0}+\int_{0}^{t} a(s, x(\cdot)) d s+\int_{0}^{t} A(s, x(\cdot)) d w(s)
$$

has a solution on $[0, l]$. This means that there exist a probabilistic measure $\mu$ on $\left(C^{0}\left([0, T], \mathbb{R}^{n}\right)\right.$ and a Wiener process in $\mathbb{R}^{n}$ given on $\left(C^{0}\left([0, T], \mathbb{R}^{n}\right), \mathcal{F}, \mu\right)$ and adapted to $\mathcal{P}_{t}$ such that the coordinate process $\xi(t)$ on $\left(C^{0}\left([0, l], \mathbb{R}^{n}\right), \mathcal{F}, \mu\right)$ and $w(t)$ satisfy (14). Together with (10) - (13), this completes the proof of Theorem.

Acknowledgements. The research is supported in part by RFBR Grant 18-01-00048.

\section{References}

1. Nelson E. Derivation of the Schrödinger Equation from Newtonian Mechanics. Physical Review, 1966, vol. 150, pp. 1079-1085. DOI: 10.1103/PhysRev.150.1079

2. Nelson E. Dynamical Theory of Brownian Motion. Princeton, Princeton University Press, 1967.

3. Nelson E. Quantum Fluctuations. Princeton, Princeton University Press, 1985.

4. Azarina S.V., Gliklikh Yu.E. Differential Inclusions with Mean Derivatives. Dynamic Systems and Applications, 2007, vol. 16, pp. 49-71.

5. Azarina S.V., Gliklikh Yu.E. Stochastic Differential Equations and Inclusions with Mean Derivatives Relative to the Past. International Journal of Difference Equations, 2009, vol. 4, no. 1 , pp. 27-41.

6. Parthasarathy K.R. Introduction to Probability and Measure. N.Y., Springer, 1978. DOI: $10.1007 / 978-1-349-03365-2$

7. Azarina S.V, Gliklikh Yu.E., Obukhovskii A.V. Solvability of Langevin Differential Inclusions with Set-Valued Diffusion Terms on Riemannian Manifolds. Applicable Analysis, 2007, vol. 86, no. 9, pp. 1105-1116. DOI: 10.1080/00036810701556045

8. Gliklikh Yu.E. Global and Lochalic Analysis with Applications to Mathematical Physics. London, Springer, 2011.

9. Deimling K. Multivalued Differential Equations. Berlin, Walter de Gruyter, 1992. DOI: $10.1515 / 9783110874228$

10. Kamenskii M., Obukhovskii V., Zecca P. Condensing Multivalued Maps and Semilinear Differential Inclusions in Banach Spaces. Berlin, Walter de Gruyter, 2001. DOI: $10.1515 / 9783110870893$ 
11. Gihman I.I., Skorohod A.V. Theory of Stochalic Processes. N.Y., Springer, 1979. DOI: $10.1007 / 978-1-4612-6202-2$

12. Gliklikh Yu.E., Obukhovskii A.V. On a Second Order Differential Inclusion with Random Perturbation of Velocity. Proceedings of Voronezh State University, 2003, no. 1, pp. 93-95.

13. Gliklikh Yu.E., Makarova A.V. On Stochastic Differential Inclusions with Current Velocities. Journal of Computational and Engineering Mathematics, 2015, vol. 2, no. 3, pp. 25-33.

Received February 28, 2019

УДК 519.216

DOI: $10.14529 / \mathrm{mmp} 190212$

\section{СТОХАСТИЧЕСКИЕ ВКЛЮЧЕНИЯ С ПРОИЗВОДНЫМИ В СРЕДНЕМ СПРАВА, ИМЕЮЩИЕ РАЗЛОЖКИЫЕ ПРАВЫЕ ЧАСТИ}

$\boldsymbol{A}$. B. Макарова, Военный учебно-научный цента ВВС «Академия имени профессора Н.Е. Жуковского и Ю.А. Гагарина», г. Воронеж,

Российская Федерация

Получена теорема существования решений для стохастических дифференциальных включений, заданных в терминах производных в среднем справа (дающих информацию о сносе) и квадратичных производных в среднем (дающих информацию о коэффициенте диффузии). Правые части, как в части с производными справа, так и в части с квадратичными производными, многозначны и полунепрерывны снизу, но не обязательно выпуклозначные. Вместо этого мы предполагаем, что они разложимы. Такие включения возникают во многих моделях физических процессов.

Ключевые слова: производные в среднем; разложимые многозначные отображения; дифференииальные включения.

Алла Викторовна Макарова, кандидат физико-математических наук, преподаватель, Военный учебно-научный центр ВВС «Академия имени профессора Н.Е. Жуковского и Ю.А. Гагарина» (г. Воронеж, Российская Федерация), allagm@mail.ru.

Поступила в редакиию 28 февраля 2019 г.

Вестник ЮУрГУ. Серия «Математическое моделирование 